\title{
Preliminary Results of Numerical Simulation of Slug Flow in a Regular T-Junction
}

\author{
Minh Tran ${ }^{1}$, Zeeshan Memon ${ }^{1}$, William Pao ${ }^{1, *}$, and Fakhruldin M Hashim ${ }^{1}$ \\ ${ }^{1}$ Universiti Teknologi PETRONAS, 32610 Seri Iskandar, Perak, Malaysia
}

\begin{abstract}
Excessive liquid carryover in T-junction due to splitting nature of two-phase flow causes serious issues in piping system. A threedimensional numerical model is employed to investigate two-phase flow split behavior of slug flow in a circular-section regular T-junction with $0.0752 \mathrm{~m}$ diameter. The Volume of Fraction method combined with k- $\varepsilon$ turbulence model and initial sinusoidal perturbation in ANSYS FLUENT is adopted to characterize the effect of slug flow behavior on the phase separation efficiency. The preliminary result reveals that the simulation work can study slug flow split in great detail and the slug characteristic plays an important role in understanding split behavior.
\end{abstract}

\section{Introduction}

T-junctions are popular component of pipeline system in the oilfield. A gas-liquid flow is likely to split into uneven flows when going to a branching $\mathrm{T}$-junction. This characteristic raises perspective of using T-Junction as a partial phase separator.

Via early experiments, the flow pattern at the main arm makes a huge impact on phase split behavior in T-junction [1-3]. Recently, same results was drawn when working with annular and slug flow and it is noticed that phase separation highly depends on the flow patterns [4]. As a result, different flow patterns should be treated in different ways to describe numerically the flow situations, such as using Lagrangian-Eulerian coupled framework for annular flow [5] and using Eulerian multiphase model for bubbly flow [6]. In case of slug flow, although it is very common in practice, few investigations have been devoted to address its separation behavior due to its nature of complexity. Therefore, the present work considers the split of slug flow which is fully-developed before approaching the junction.

From experiment, by decreasing gas velocity or increasing liquid velocity, less liquid carryover is formed [7]. This idea was confirmed by a numerical study on two-phase stratified flow separation in T-junctions with different diameter ratios [8]. The combination of experiment and numerical investigation shows a great potential to understand phase split behavior and solve liquid carryover problems. At the same time, working with wet-gas separation in T-junction, mass split ratio is one of the most influential factors in phase separation [9]. In experiments, the mass split ratio is controlled by valves at the run arm, while in the simulation work, it is featured by the outflow boundary condition [10].

* Corresponding author: william.pao@utp.edu.my 


\section{Numerical model}

\subsection{Geometry domain and meshing}

To simulate the T-junction, the slug flow is developed in the main arm of $6 \mathrm{~m}$ in length from a horizontal inlet for gas and an upward inlet for water (Fig. 1). All arms have the same diameter of $0.0752 \mathrm{~m}$. Based on above experimental setup, the geometry is produced and meshed by ANSYS ICEM to retain high solver accuracy with optimum number of hexahedral elements. Fig. 3 illustrates unstructured mesh of T-junction domain. In order to simulate in FLUENT, meshing statistics must meet some quality measure requirement [11].

A grid independence test is required to achieve a grid-independent solution. The test is carried out with total 10 simulation cases with a different number of hexahedral elements characterized by maximum element size in wall boundaries from the finest of $6 \mathrm{~mm}$ to the coarsest of $30 \mathrm{~mm}$. The average total pressure and average air fraction in the branch outlet plane were used as convergent criteria. Fig. 2 shows a clear trend that when the pressure and air fraction at branch reach convergence, finer meshing below $12 \mathrm{~mm}$ does not improve the accuracy.

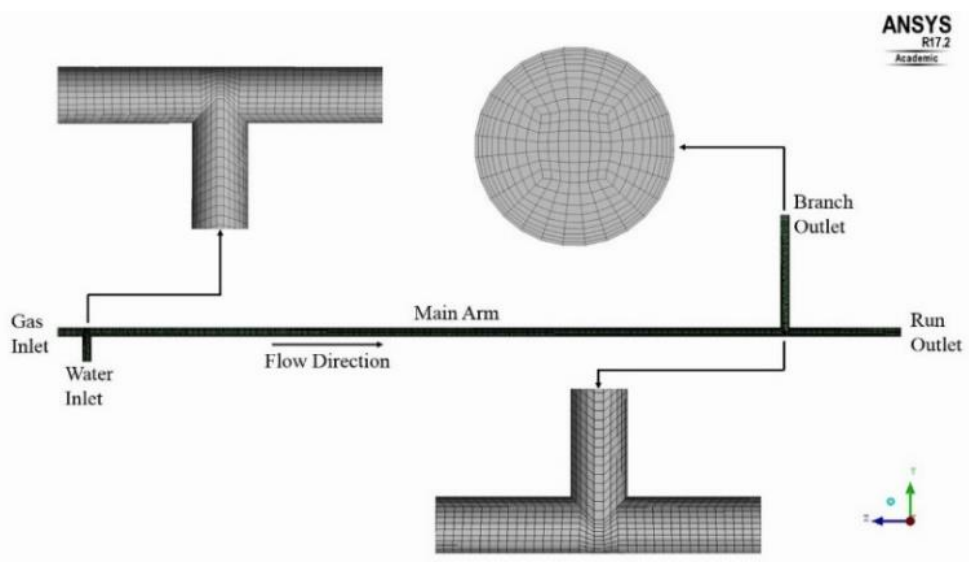

Fig. 1. Modeled T-junction geometry

\subsection{Multiphase model}

According to ANSYS Fluent Theory Guide [12], different methods should be applied for different flow regimes generated. Volume of Fraction method (VOF) is recommended to model slug flow. To describe air-water interaction, both phases share only one set of conservation equations and VOF method enhance the tracking of volume fraction in every cell within computational domain. Besides, another setup is a combination of Pressure Implicit with Split Operator algorithm (PISO) for pressure-velocity coupling, the first-order upwind scheme for discretization of mass, momentum, volume fraction, turbulent kinetic energy and turbulent dissipation rate equations. In addition, a time step is 0.0001 with the maximum 20 iterations each. The convergence criterion for all variables is $10^{-5}$.

The primary phase is air. Superficial velocity of air and water used in dependency test is $2.75 \mathrm{~m} / \mathrm{s}$ and $1.5 \mathrm{~m} / \mathrm{s}$ respectively under the temperature of $298 \mathrm{~K}$, and operating pressure of $101,325 \mathrm{~Pa}$. Initially, the main pipe is filled up with both air and water. User Defined Functions in ANSYS FLUENT is employed to generate sinusoidal perturbation of phase interface as initialization. Both mean air and water volume fraction is 0.5 with a water level 
function (equation 1). The interface formulation is based on length of slug observation in the experiment of Saieed [10] and also slug flow simulation in the horizontal pipe from Frank's work [13]. In the transient mode, this preset sinusoidal initial condition speeds up the simulation to quickly transform the slug flow before reaching the junction.

$$
y=y_{0}+0.25 D \sin \left(2 \pi \frac{v_{l} z}{0.25 L}\right)
$$

where $\mathrm{y}_{0}=0, \mathrm{D}$ and $\mathrm{L}$ are main pipe diameter and length in $\mathrm{m}$, respectively, and $v_{1}$ is superficial water velocity in $\mathrm{m} / \mathrm{s}$.
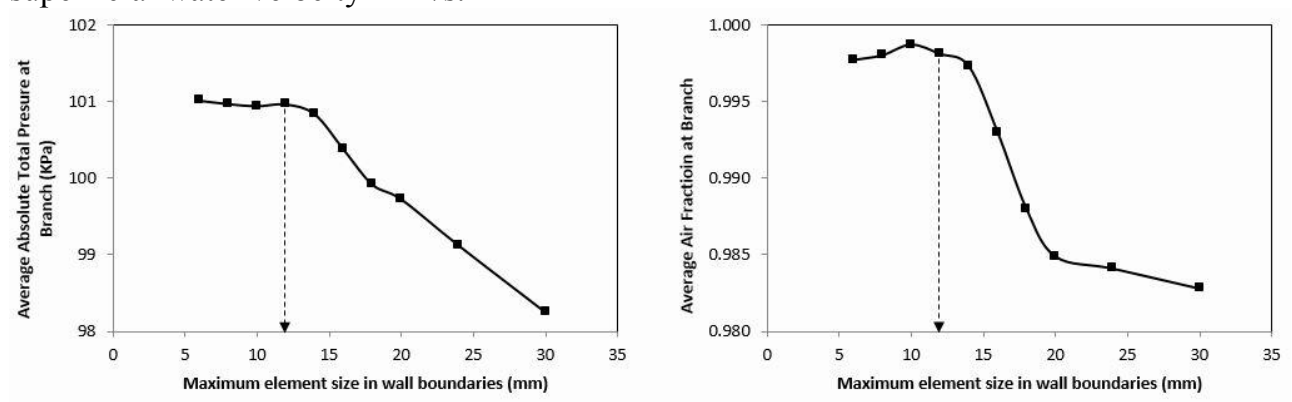

Fig. 2. Solution convergence study in 10 different meshing cases

\subsection{Governing equations}

The governing equations used in this study are stated as:

- Continuity Equation:

$$
\frac{\partial}{\partial t}(\rho)+\nabla \cdot(\rho \vec{v})=0
$$

- Momentum Balance Equation:

$$
\frac{\partial}{\partial t}(\rho \vec{v})+\nabla \cdot(\rho \vec{v} \vec{v})=-\nabla P+\nabla \overline{\bar{\tau}}+\rho_{g} \vec{g}+\vec{R}_{l, g}
$$

Here, $\rho$ is the density, $v$ is the velocity, $P$ is the combined pressure for the two phases, $g$ is the gravitation acceleration, $\tau$ is the tensor term of stress-strain, subscripts $g$ and 1 stands for gas and liquid, respectively, and $\mathrm{R}$ is the interaction force at the interface of gas and liquid.

- Turbulence Equation

The governing equations in the k- $\varepsilon$ model are shown as:

$$
\begin{gathered}
\frac{\partial\left(\rho_{m} k\right)}{\partial t}+\nabla \cdot\left(\rho_{m} k \overrightarrow{v_{m}}\right)=\nabla \cdot\left(\frac{\mu_{t, m}}{\sigma_{k}} \nabla k\right)+G_{k, m}-\rho_{m} \varepsilon \\
\frac{\partial\left(\rho_{m} \varepsilon\right)}{\partial t}+\nabla \cdot\left(\rho_{m} \varepsilon \overrightarrow{v_{m}}\right)=\nabla \cdot\left(\frac{\mu_{t, m}}{\sigma_{k}} \nabla \varepsilon\right)+\frac{\varepsilon}{k}\left(C_{1 \varepsilon} G_{k, m}-C_{2 \varepsilon} \rho_{m} \varepsilon\right)
\end{gathered}
$$

where the mixture density, velocity, and turbulent viscosity are expressed as:

$$
\begin{gathered}
\rho_{m}=\alpha_{l} \rho_{l}+\alpha_{g} \rho_{g} \\
\vec{v}_{m}=\frac{\alpha_{l} \rho_{l} v_{l}+\alpha_{g} \rho_{g} v_{g}}{\alpha_{l} \rho_{l}+\alpha_{g} \rho_{g}} \\
\mu_{t, m}=C_{\mu} \rho_{m} \frac{k^{2}}{\varepsilon}
\end{gathered}
$$




\section{Results and discussion}

\subsection{Slug flow development}

The slug flow developed in the main pipe is validated with other numerical results (Fig. 3) [14-16]. Slug flow is the flow in which gas bubbles and elongated gas bubbles move along the upper part of the pipe [17]. According to this explanation, Schepper and Rahimi's slug flow model showed poor results with no elongated gas bubbles. Meanwhile, Ban's slug flow presents elongated bubbles but lack of small gas bubbles along the moving direction. With VOF enhancing tracking precise interface between air and water together with sinusoidal perturbation initialization, the present model illustrates the unsteadiness of slug behavior generating slug jump to plug the pipe section and it travels along the pipe with both elongated and small gas bubbles at the upper part of the pipe.

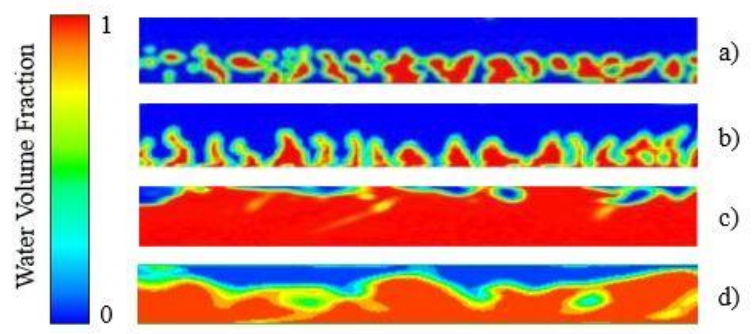

Fig. 3. Comparison of present slug flow model (d) with other models a) De Schepper [14] b) Rahimi [15] c) Ban [16]

\subsection{Validation of phase separation with experimental data}

For validation purpose, experimental data of Saieed [10] is chosen as phase separation benchmark. Due to the limited capacity of equipment in his experiment, the range of air and water superficial velocities are $0.648-1.4 \mathrm{~m} / \mathrm{s}$ and $0.21-0.84 \mathrm{~m} / \mathrm{s}$, respectively. In present model, air and water velocity are $0.648 \mathrm{~m} / \mathrm{s}$ and $0.84 \mathrm{~m} / \mathrm{s}$. It is evident that the phase separation obtained from this validation analysis is in a reasonable agreement with the benchmarks (Fig. 4). Therefore, the present model is able to be used for further investigation.

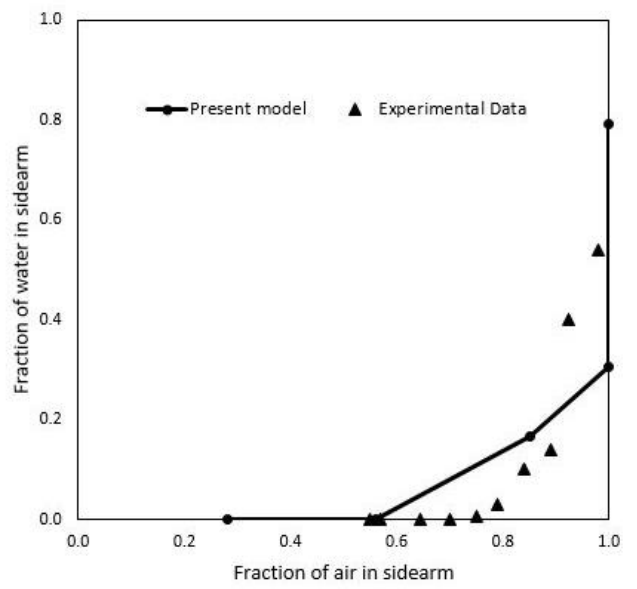

Fig. 4. Air and water fraction in present model validated with experimental data [10]

\subsection{Phase separation behavior}


Behavior of the slug flow split is presented (Fig. 5). Before the slug reaches the junction, there is a negligible amount of water sucked into the branch arm. When the slug hits the junction, water starts to enter the branch, creating a dividing streamline between air and water. A clear view of dividing streamline is shown using velocity vector (Fig. 6). This phenomenon is also observed in Liu and Li's simulation model of bubbly flow [6]. The gas diverts faster into the run arm through the left side of the dividing streamline. Additionally, Figure 8 confirms the idea of backflow region in the sidearm, which was discussed in Popp and Sallet's experiment [18]. The difference between air and water inertia is attributed to for this phenomenon.

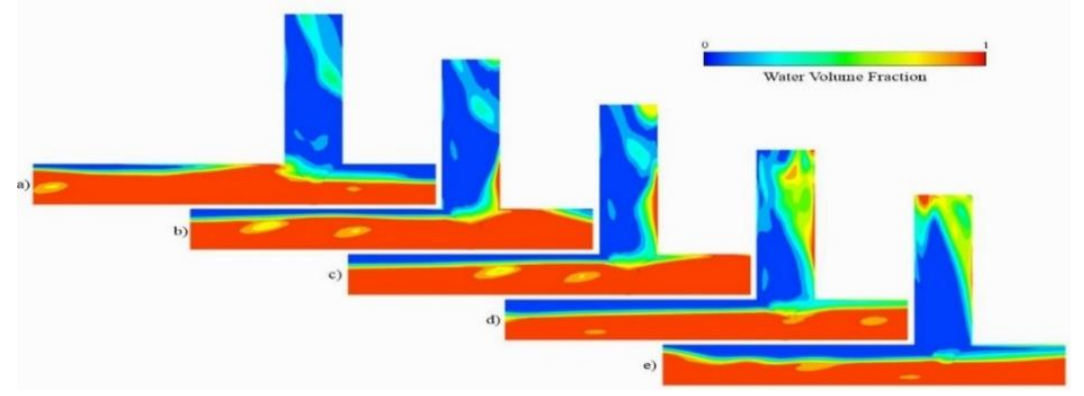

Fig. 5. Contours of slug flow split behavior at the junction from $2.0 \mathrm{~s}$ to $2.5 \mathrm{~s}$ with description a) Slug before reaching the junction b) Slug hitting the junction c) Slug blocking run arm d) More water going to branch e) New slug reaching the junction

Moving to the next description in Fig. 5, the slug tends to block the entire section in the run arm after passing the junction. This blocking increases pressure in the run arm, thus water is more likely to run into the branch due to higher difference pressure between the main arm and the branch arm. This continues as the main force causing the liquid carryover until the slug blocking in the run arm releases. Then, another slug in the main arm is going to reach the junction ad starts another period which is described above. Therefore, longer slug length decreases the frequency of slug hitting the junction, leading to less water running into the branch due to blocking run arm section.

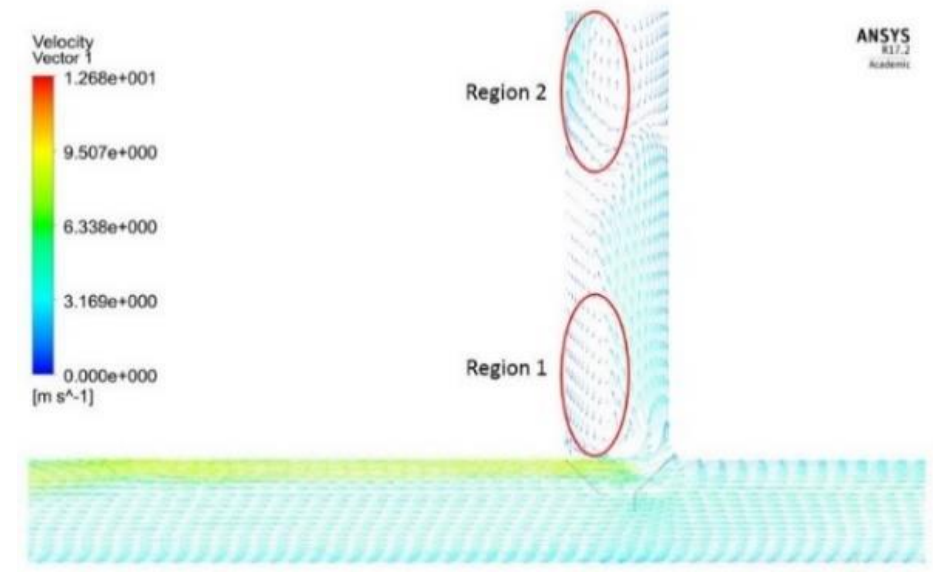

Fig. 6. Velocity vector at the junction

Further investigation in velocity vector (Fig. 6) shows two mechanisms affecting the flow behavior in the sidearm. The first region is dominated by gravity force, which is named as 
backflow. A part of slug jump falls back to the mainstream and the rest reaches the region 2 . At the same time, the upper part of the sidearm is mostly taken place by air, which creates a pressure drop lifting the water at the region 2.

\section{Conclusions}

A numerical study using Volume of Fraction method and sinusoidal perturbation is carried out to investigate slug flow behavior in a regular T-junction. The phenomenon of dividing streamline and pressure increase at the junction due to slug blocking run arm is emphasized as the main causes for liquid carryover. Based on this finding, the effect of slug flow characteristics such as slug length and slug frequency, on phase separation should be studied in more details to improve phase split efficiency.

The authors would like to acknowledge the funding by Ministry of Higher Education through FRGS 0153AB-L03 and PETRONAS through YUTP Fundamental Research Grant 0153AA-E03.

\section{References}

1. O. Shoham, J.P. Brill, Y. Taitel, Chem. Eng. Sci. 42 (1987) 2667-2676.

2. E. Wren, Geometric Effects in Phase Split at a Large Diameter T-junction (University of Nottingham, 2001)

3. G. Baker, Separation and Control of Gas-Liquid Flows at Horizontal T-Junctions, (University of Nottingham, 2003)

4. K. He, S. Wang, J. Huang, Int. J. Heat and Mass Trans. 54 (2011) 3587-3593.

5. D. Adechy, R.I. Issa, Computers \& Fluids 33 (2004) 289-313.

6. Y. Liu, W.Z. Li, Int. J. Air-Cond. Ref. 19 (2011) 253-262.

7. G. Das, P.K. Das, B.J. Azzopardi, Int. J. Multiphase Flow 31 (2005) 514-528.

8. W. Pao, A. Saieed, F.M. Hashim, R.B.M. Norpiah, Materialwissenschaft Und Werkstofftechnik 48 (2017) 255-260.

9. W. Pao, F.M. Hashim, L.H. Ming, MATEC Web of Conferences 13 (2014) 03009.

10. A. Saieed, Two-Phase Separation in a T-Junction (Universiti Teknologi PETRONAS, 2017)

11. ANSYS Fluent Meshing User's Guide (2013).

12. ANSYS Fluent Theory Guide (2016).

13. T. Frank, Proceedings of the 11th International Topical Meeting on Nuclear Reactor Thermal-Hydraulics (NURETH-11), Avignon, France, October, 2005, pp. 2-6.

14. S.C.K. De Schepper, G.J. Heynderickx, G.B. Marin, Chem. Eng. J. 138 (2008) 349357.

15. R. Rahimi, Gas Processing 1 (2013) 70-87.

16. S. Ban, Numerical Simulation of Two-Phase Slug Flow Regime in Horizontal Pipe (Universiti Teknologi PETRONAS, 2017)

17. O. Baker, Fall Meeting of the Petroleum Branch of AIME (Society of Petroleum Engineers, 1953)

18. M. Popp, D.W. Sallet, (1983) 67-88. 